and, therefore, they are attractive diagnostic and prognostic markers. Mewar et al. initially identified the ferritin heavy-chain polypeptide as a potential target of RA autoantibodies in vitro, and then evaluated antiferritin antibody levels in patients with RA and a series of control populations.

The incidence of antiferritin antibodies was significantly higher in individuals with RA than in the control groups: $16.4 \%(n=366)$ with established RA; $19 \%(n=118)$ with early RA; $2.1 \%(n=94)$ with osteoarthritis; $2.1 \%(n=97)$ with systemic lupus erythematosus; and $2.7 \%(n=73)$ of healthy blood donors were positive for antiferritin antibodies. Hence antiferritin antibodies, though having a relatively low sensitivity of $17 \%$, were highly specific (>97\%) in these patient groups for RA.

The presence of antiferritin antibodies was associated with male sex. Antibody levels were associated with disease severity, but not with disease duration, indicating that they were not simply a consequence of longstanding disease. These findings suggest that antiferritin antibodies might contribute to joint damage, and could potentially be used as diagnostic or prognostic markers of RA. Further studies of antiferritin antibodies in RA are warranted.

Rachel Murphy

Original article Mewar D et al. (2005) Antiferritin antibodies discovered by phage display expression cloning are associated with radiographic damage in rheumatoid arthritis. Arthritis Rheum 52: 3868-3872

\section{Long-term steroids might not increase lymphoma risk for some patients}

Some studies have suggested that long-term steroid use doubles a patient's risk of lymphoma; however, many of the inflammatory conditions currently treated with steroids or other potentially lymphoma-inducing drugs themselves carry an increased lymphoma risk. A new, large, population-based case-control study, using prospectively recorded data from the Swedish Cancer Register, aimed to avoid these confounding factors by examining lymphoma risk in patients with polymyalgia rheumatica (PMR) and giant cell arteritis (GCA), conditions that are uniformly and successfully treated with 1-2 years of steroids as single immunosuppressive therapy.

In all, 42,676 lymphoma cases and 78,487 matched controls were identified. Of these, 153 cases and 345 controls had been hospitalized with PMR or GCA at least 1 year before diagnosis of the index lymphoma case, corresponding to an unexpected and statistically significant reduction in lymphoma risk of $19 \%$ (odds ratio 0.81; 95\% Cl 0.67-0.98). Lymphoma risk associated with GCA was slightly lower than for PMR.

These results imply that daily oral corticosteroid use, in moderate to high cumulative doses over 1-3 years, is not associated with increased occurrence of lymphoma, say the authors, although it might not be appropriate to generalize these results to all conditions in which steroids are used.

Caroline Barranco

Original article Askling J et al. (2005) Do steroids increase lymphoma risk? A case-control study of lymphoma risk in polymyalgia rheumatica/giant cell arteritis. Ann Rheum Dis 64: $1765-1768$

\section{Oral PPAR $\gamma$ agonist THR9021 reduces the severity of collagen-induced arthritis}

Peroxisome proliferative-activated receptor (PPAR) $\gamma$ agonists are used to treat diabetes, but are known to have anti-inflammatory and immunomodulatory effects that are being evaluated in a range of inflammatory conditions. Tomita et al. have shown that oral THR9021, a novel PPAR $\gamma$ agonist, reduces the severity of collagen-induced arthritis (CIA) in mice, via inhibition of proinflammatory cytokine production, T-cell proliferation, and osteoclastogenesis.

Treatment with oral THR9021 started on day 21 after induction of CIA. Treated and control mice with CIA were harvested on day 42; THR9021 delayed the onset and greatly reduced the severity of arthritis compared with controls. Treated mice had markedly reduced spleen-cell proliferation and markedly reduced production of the proinflammatory cytokines tumor necrosis factor, interleukin-1 $\beta$ and interferon $\gamma$ at both local and systemic levels, compared with controls. Levels of these cytokines approached those in mice without CIA. THR9021 inhibited the differentiation of osteoclasts and suppressed production of MCP-1, a cytokine responsible for monocyte recruitment and macrophage activation; this might be the most important of its effects, say the authors.

Interestingly, these beneficial effects were seen at a dose 10 times lower than that used 
in a similar study of troglitazone, despite THR9021's weaker activity as a PPAR $\gamma$ agonist. The correspondingly weaker adipogenicity and oral formulation of THR9021 might provide additional clinical benefits.

\section{Caroline Barranco}

Original article Tomita T et al. (2006) THR0921, a novel peroxisome proliferator-activated receptor gamma agonist, reduces the severity of collagen-induced arthritis. Arthritis Res Ther 8: R7

\section{ANCA-associated small vessel vasculitis: treatment with mycophenolate mofetil}

The immunosuppressant mycophenolate mofetil (MMF) could provide an effective alternative to combined corticosteroids and cytotoxic agents in the treatment of antineutrophil cytoplasmic autoantibody-associated small vessel vasculitis (anca-svv). Joy et al. report that MMF obviates the need for cytotoxic drugs and avoids some of the risks associated with long-term use of the combined regimen, such as secondary malignancies, nephrotoxicity, and reduced fertility.

This small, dose-escalating pilot study enrolled patients with non-life-threatening ANCA-SVV who had relapsing disease $(n=6)$ or who had failed to respond to previous cyclophosphamide treatment $(n=6)$. MMF was administered twice daily from the first study day, with the target dose of 1,000-1,500 mg twice daily administered for 24 weeks. Concomitant corticosteroids were permitted. From weeks 24 to 52, other agents could be used to replace or bolster MMF treatment.

As measured by the BVAS, disease activity significantly decreased with MMF induction treatment between baseline and week 24 $(P=0.0013)$, and between baseline and week $52(P=0.0044)$. Recurrent use of a cytotoxic agent was avoided in 10/12 patients. Some disease activity remained, however, and only a minority of patients achieved a long-lasting remission, while several patients responded poorly to treatment. Interestingly, all patients who showed an early and sustained decrease in disease activity were in the relapsing group, indicating that MMF is ineffective in those resistant to cyclophosphamide induction therapy.

Joy et al. recommend that therapy is tailored to each individual's disease course, and suggest that combination therapy is likely to be most effective in controlling disease and limiting toxicity. Further investigations into MMF treatment are needed.

Pippa Murdie

Original article Joy MS et al. (2005) A pilot study using mycophenolate mofetil in relapsing or resistant ANCA small vessel vasculitis. Nephrol Dial Transplant 20: 2725-2732

\section{Rheumatoid arthritis patients' participation in clinical trials}

Inclusion of a heterogeneous study population is desirable in clinical trials; however, accruing adequate numbers from minority groups is typically difficult. A survey of patients with rheumatoid arthritis (RA) has recently been conducted, with the aim of elucidating the factors that affect patients' decisions on whether to participate in studies.

Researchers asked 191 RA patients if they would participate in the survey, of whom 144 patients agreed; 57\% were Hispanic, 25\% Caucasian, 12\% Asian and 6\% African American. Most patients were of a low socioeconomic background. Patients answered a questionnaire that gathered information on demographics, health status and important factors when considering participation in clinical trials.

Advantages of participating in a clinical trial included the opportunity to help others, the possibility of improved health, early access to new treatments, and availability of free treatment. In general, there were no significant differences between Caucasians and Hispanics for weighting of importance. The disadvantages were unknown side effects, the need to stop current therapy, lack of trust in doctors and the need to travel to research sites. Caucasians were more likely to decline trial participation because of negative factors than Hispanics, indicating that low accrual of Hispanics to clinical trials is because of a lack of awareness of potential benefits. Most patients in each ethnic group indicated they would be willing to participate in a clinical study, apart from Asians, among whom only $31 \%$ agreed.

The authors conclude that consideration of ethnic-specific factors during trial recruitment could facilitate inclusion in clinical trials.

\section{Rachel Murphy}

Original article Lee SJ et al. (2005) Factors affecting rheumatoid arthritis patients' decisions to participate in clinical trials. J Rheumatol 32: 2317-2325 